\title{
Pacific
}

Journal of

Mathematics

\section{THE TEMPERLEY-LIEB ALGEBRA AT ROOTS OF UNITY}

Frederick Michael Goodman AND Hans Wenzl 


\title{
THE TEMPERLEY-LIEB ALGEBRA AT ROOTS OF UNITY
}

Frederick M. Goodman ANd HaNs WenZl

\begin{abstract}
We present general techniques to determine the structure of Hecke algebras and similar algebras in the non-semisimple case. We apply these to give a complete description of the structure of the TemperleyLieb algebras at a root of unity. Our description implies in particular that the representation of these algebras on tensor space $\left(C^{2}\right)^{\otimes n}$ is faithful.
\end{abstract}

Introduction. We shall consider ascending sequences of finite dimensional algebras $A_{1} \subset A_{2} \subset \cdots$, given by generators and relations, where the relations depend on one or several parameters. Moreover,we also assume that the discriminants of these algebras are non-zero polynomials or rational functions in the parameters. This means the algebras are semisimple except for special values of the parameters. For applications (to the construction of topological invariants, the construction of subfactors, or in statistical mechanical models) these algebras are often needed at the critical values of the parameters; in such cases, interesting semisimple quotients have been constructed in [J1], [W2,4].

In this paper, we initiate a systematic study of the structure of such algebras at the critical values of the parameters, where they are not semisimple.

For the examples we have in mind such as Hecke algebras, Brauer algebras, etc., the structure is known in the semisimple case and can be described by the Bratteli diagram, which encodes how an irreducible representation of $A_{n}$, restricted to $A_{n-1}$, decomposes into irreducible representations of $A_{n-1}$. If all the multiplicities in the decompositions are 0 or 1 , one can use this to define special path idempotents and matrix units (see e.g. [SV], [W1,2], [RW]), labelled by paths on the Bratteli diagram. These matrix units are only well defined for generic values of the parameters, for which the algebras are semisimple. Nevertheless, their (usually inductive) defining formulas carry a lot of information about the structure of the algebras which can also be exploited at the critical parameter values. In more detail, our main techniques are as follows: 
1. Evaluation principle: We use representations of the algebras to obtain algebraic identities with the parameters viewed as indeterminates. Although these representations are not necessarily well defined at a critical value of the parameters, nevertheless the identities remain valid as long as the quantities appearing in them are well-defined at the critical value.

2. Spectral analysis for path idempotents: Let us assume for simplicity that the algebra $A_{n}$ can be obtained from $A_{n-1}$ by adding one more generator, say $g_{n-1}$. (We assume all the $A_{n}$ 's to be subalgebras of their inductive limit.) Moreover, assume the path idempotents for $A_{n-1}$ are known. Then it is possible in interesting examples to obtain the path idempotents for $A_{n}$ by determining the "spectral projections" of $p_{s} g_{n-1} p_{s}$, where $p_{s}$ is a path idempotent of $A_{n-1}$ (see e.g. [W1,2], [RW]). In these examples at generic parameter values, the characteristic polynomials of such elements have simple roots only; for the critical values, however, some of the characteristic values may coincide. This basically means that one can no longer "diagonalize" these elements; instead, one has to deal with Jordan forms, which yield nilpotent elements in the radical.

3. Regular idempotents: In our examples, although many path idempotents cease to be well defined at the critical parameter values, one still can find "good" path idempotents (or sometimes sums of path idempotents) for each component of the maximal semisimple quotient.

These principles have been stated quite generally. We expect that they can be applied to Iwahori-Hecke algebras at roots of unity, to Brauer algebras [Br, W3] and their $q$-deformations, the so-called Birman-Wenzl algebras [BW, W4] and presumably also to the group algebras of the symmetric group in positive characteristic.

As a first example, these techniques will be applied here to the Temperley-Lieb algebras, which are quotients of the Iwahori-Hecke algebras defined by generators $e_{i}, i=1,2, \ldots, n-1$, and relations $e_{i} e_{i \pm 1} e_{i}=q /(1+q)^{2} e_{i}$ and $e_{i} e_{j}=e_{j} e_{i}$ if $|i-j| \geq 2$. These algebras first appeared in work of $\mathrm{H}$. Temperley and $\mathrm{E}$. Lieb analyzing certain statistical mechanical models [TL, B]. They were rediscovered by V. Jones in connection with his study of von Neumann subfactors and used by him to define the Jones link invariant [J1,2].

It is well known that for $q$ not a root of unity the Temperley-Lieb algebra $A_{n}(q)$ is isomorphic to the quotient of the group algebra of the symmetric group whose irreducible representations are labelled by 
partitions, or Young diagrams, with at most two parts. In particular, the simple component labelled by the diagram $\lambda$ is isomorphic to the $f_{\lambda}$-by- $f_{\lambda}$ matrix ring, where $f_{\lambda}$ is the number of standard tableaux of shape $\lambda$.

We can give a similar description of $A_{n}(q)$ for $q$ a primitive $l$ th root of unity, $l \geq 3$. We associate to a diagram $\lambda=\left[\lambda_{1}, \lambda_{2}\right]$ its weight $w(\lambda)=\lambda_{1}-\lambda_{2}+1$. A diagram is called critical if its weight is divisible by $l$. Let the affine reflection group $A_{1}^{(1)}$ act on $\mathbf{Z}$ via reflections about the numbers $m l, m \in \mathbf{Z}$; this also defines a partial action on diagrams with a fixed size, via their weights. The results are then:

1. The dimension of $A_{n}(q)$ is the Catalan number $\frac{1}{n+1}\left(\begin{array}{c}2 n \\ n\end{array}\right)$, as for generic values of $q$.

2. The blocks of $A_{n}(q)$ are labelled by the critical diagrams of size $n$ and by the orbits of the action of $A_{1}^{(1)}$ on the non-critical diagrams of size $n$.

3. The blocks labelled by critical diagrams are simple with the same dimension as for generic values of $q$.

4. For a block labelled by a non-critical orbit, the simple components in the maximal semisimple quotient are again labelled by the individual diagrams in the orbit. If $\mu$ is a diagram with weight $m l<w(\mu)<(m+1) l$, then the dimension of the corresponding simple component is $\left(f_{\mu}^{L}\right)^{2}$, where $f_{\mu}^{L}$ is the number of standard tableaux of shape $\mu$ which (when regarded as increasing sequences of Young diagrams) have their last critical diagram of weight $\mathrm{ml}$.

If the orbit $[\lambda]$ is $\left\{\lambda^{(0)}, \lambda^{(1)}, \ldots, \lambda^{(k)}\right\}$, where $\lambda^{(m)}$ has weight $m l<w\left(\lambda^{(m)}\right)<(m+1) l$, then one has the formula

$$
f_{\lambda^{(m)}}^{L}=\sum_{j \geq m}(-1)^{j-m} f_{\lambda^{(j)}}
$$

5. The block corresponding to a non-critical orbit [ $\lambda]$ contains an isomorphic lifting of the maximal semisimple quotient of the block, with minimal central idempotents $z_{\mu}^{L}$ for $\mu \in[\lambda]$. If the weight of $\mu$ is less than $l$, then $z_{\mu}^{L} A_{n}(q) z_{\mu}^{L} \cong \mathrm{M}_{f_{\mu}^{L}}$, where $\mathbf{M}_{k}$ denotes the $k$-by- $k$ matrix ring. Otherwise $z_{\mu}^{L} A_{n}(q) z_{\mu}^{L} \cong \mathbf{M}_{f_{\mu}^{L}} \otimes \mathbf{C}[x] /\left(x^{2}\right)$.

The radical of the block is nilpotent of exponent 3 , and is spanned by the spaces $z_{\mu}^{L} A_{n}(q) z_{\nu}^{L}$ for diagrams $\mu, \nu \in[\lambda]$ which are in adjacent fundamental intervals for the $A_{1}^{(1)}$ action, and by the algebra $\operatorname{rad}\left(z_{\mu}^{L} A_{n}(q) z_{\mu}^{L}\right)$. If $\mu$ and $\nu$ are not adjacent, then $z_{\mu}^{L} A_{n}(q) z_{\nu}^{L}$ $=(0)$. 
A complete description of the multiplication rules for the radical will be given at the end of $\S 2$.

6. An important corollary of this structure theorem is that the representation of the Temperley-Lieb algebra $A_{n}(q)$ in $\operatorname{End}\left(\left(\mathbf{C}^{2}\right)^{\otimes n}\right)$ is faithful, and the image has dimension $C_{n}=\frac{1}{n+1}\left(\begin{array}{c}2 n \\ n\end{array}\right)$, also when $q$ is a root of unity.

After this work was completed, we learned that P. Martin had obtained the same results independently $[\mathbf{M}]$; however, our emphasis is somewhat different, and we believe that there are some advantages to our exposition. We would like to thank Martin for discussing his work with us.

\section{Preliminaries.}

0.1. Finite dimensional semisimple algebras. We first need some notation for the combinatorial description of increasing sequences of finite dimensional complex semisimple algebras. Let $\mathbf{M}_{n}$ denote the ring of all complex $n \times n$ matrices. If $A$ and $B$ are semisimple complex algebras, we can write them as $A=\bigoplus A_{\lambda}$ and $B=\bigoplus B_{\mu}$ with $A_{\lambda} \cong \mathrm{M}_{a_{\lambda}}$ and $B_{\mu} \cong \mathrm{M}_{b_{\mu}}$ for appropriate natural numbers $a_{\lambda}$ and $b_{\mu}$. The vector $\vec{b}$ whose components are the numbers $b_{\mu}$ is called the dimension vector of $B$.

If $A$ is a subalgebra of $B$, any simple $B_{\mu}$-module is an $A$-module. Let $g_{\lambda \mu}$ be the number of simple $A_{\lambda}$-modules in its decomposition into simple $A$-modules. The matrix $G=\left(g_{\lambda \mu}\right)$ is called the inclusion matrix for $A \subset B$. The inclusion of $A$ in $B$ is conveniently described by an inclusion diagram, or Bratteli diagram, which is a bipartite graph in which one set of vertices label the simple direct summands $A_{\lambda}$ of $A$, and the other set labels the simple summands $B_{\mu}$ of $B$. Then the vertex corresponding to $A_{\lambda}$ is joined with the vertex corresponding to $B_{\mu}$ by $g_{\lambda \mu}$ edges. If $A$ and $B$ have the same identity, the dimension vectors of $A$ and $B$ are related by $\vec{b}=G^{t} \vec{a}$.

The inclusion matrix can also be interpreted in the following way: Let $p_{\lambda}$ be a minimal idempotent of $A_{\lambda}$ and let $z_{\mu}$ be a minimal central idempotent in $B$, the identity of $B_{\mu}$. Then $g_{\lambda \mu}$ is the number of idempotents in any decomposition of $p_{\lambda} z_{\mu}$ into minimal idempotents in $B_{\mu}$.

0.2. The Temperley-Lieb algebra. The (abstract) Temperley-Lieb algebra $A_{n}$ is the $\mathbf{C}(x)$-algebra given by generators $e_{1}, e_{2}, \ldots, e_{n-1}$ and the following relations.

$(\mathrm{B} 1)^{\prime} e_{i} e_{i \pm 1} e_{i}=x /(1+x)^{2} e_{i}$ for all $i$. 
(B2) $e_{i} e_{j}=e_{j} e_{i}$ for $|i-j| \geq 2$.

(H) $e_{i}^{2}=e_{i}$ for all $i$.

The Temperley-Lieb algebra is a quotient of the Iwahori-Hecke algebra $H_{n}$ of type $A$; for the Hecke algebra, $(\mathrm{B} 1)^{\prime}$ is replaced by the more general relation

(B1) $e_{i} e_{i+1} e_{i}-x /(1+x)^{2} e_{i}=e_{i+1} e_{i} e_{i+1}-x /(1+x)^{2} e_{i+1}$ for $i=$ $1,2, \ldots, n-2$.

For $q \in \mathbf{C} \backslash\{0,-1\}$, the complex Temperley-Lieb algebra $A_{n}(q)$ is the $\mathrm{C}$-algebra given by generators $e_{1}, e_{2}, \ldots, e_{n-1}$ and relations obtained by substituting $q$ for $x$ in the relations $(\mathrm{B} 1)^{\prime},(\mathrm{B} 2)$, and $(\mathrm{H})$.

The complex algebra $A_{n}(q)$ is an "evaluation" of the $\mathbf{C}(x)$-algebra $A_{n}$ in the following sense. Let $\mathbf{C}[x]_{(x-q)}$ denote the localization of the polynomial ring $\mathbf{C}[x]$ at $(x-q)$, i.e. $\mathbf{C}[x]_{(x-q)}$ consists of all those rational functions whose denominators do not have a zero at $q$. Let $\left(A_{n}\right)_{q}$ be the $\mathbf{C}$-subalgebra of $A_{n}$ consisting of all $\mathbf{C}[x]_{(x-q)^{-}}$ linear combinations of products of the generators. We show that there is an evaluation homomorphism from $\left(A_{n}\right)_{q}$ onto $A_{n}(q)$ mapping generators to generators. Consequently we refer to the elements of $\left(A_{n}\right)_{q}$ as evaluable at $q$.

Proposition 0.1. Fix $q \in \mathbf{C} \backslash\{0,-1\}$.

(a) There is a homomorphism of $\left(A_{n}\right)_{q}$ onto $A_{n}(q)$ mapping $e_{i}$ to $e_{i}$ for all $i$.

(b) $\operatorname{dim}_{\mathbf{C}} A_{n}(q)=\operatorname{dim}_{\mathbf{C}(x)} A_{n}=\frac{1}{n+1}\left(\begin{array}{c}2 n \\ n\end{array}\right)$.

For $u \in\left(A_{n}\right)_{q}$, let $u(q)$ denote its image in $A_{n}(q)$ under the evaluation homomorphism.

(c) (Principle of constancy of dimension.) Let $e$ and $f$ be idempotents in $\left(A_{n}\right)_{q}$. Then

$$
\operatorname{dim}_{\mathbf{C}} e(q) A_{n}(q) f(q)=\operatorname{dim}_{\mathbf{C}(x)} e A_{n} f .
$$

Proof. Consider the free $\mathbf{C}(x)$-algebra $P=\mathbf{C}(x)\left\langle y_{1}, \ldots, y_{n-1}\right\rangle$ and let $I$ denote the ideal of $P$ generated by

$$
\left\{y_{i} y_{i \pm 1} y_{i}-x /(1+x)^{2} y_{i}\right\} \cup\left\{y_{i} y_{j}-y_{j} y_{i} \text { for }|i-j| \geq 2\right\} \cup\left\{y_{i}^{2}-y_{i}\right\} \text {. }
$$

Thus $A_{n} \cong P / I$. Likewise, let $P_{L}=\mathbf{C}[x]_{(x-q)}\left\langle y_{1}, \ldots, y_{n-1}\right\rangle$, and let $I_{L}$ denote the ideal of $P_{L}$ generated by the same set of elements. Call a word in $y_{1}, \ldots, y_{n-1}$ reduced if it is not congruent modulo $I$ to any shorter word. By the argument of [J1], 4.1.4, there is a family $F_{0}$ of reduced words such that $1 \in F_{0}$, the cardinality of $F_{0}$ is the 
Catalan number $C_{n}=\frac{1}{n+1}\left(\begin{array}{c}2 n \\ n\end{array}\right)$, and such that any word is congruent modulo $I_{L}$ to some element of $F_{0}$. Hence the image $F$ of $F_{0}$ in $A_{n}$ is a spanning set, and $\operatorname{dim}_{\mathrm{C}(x)} A_{n} \leq C_{n}$. On the other hand, by producing sufficiently many inequivalent irreducible representations of $A_{n}\left([\mathbf{J 1}], \S 5\right.$, and [W2]), one obtains that $A_{n}$ is semisimple with $\operatorname{dim}_{\mathbf{C}(x)} A_{n}=C_{n}$. Thus $F$ is a basis of $A_{n}$, and $\operatorname{span}\left(F_{0}\right) \cap I=(0)$. It follows that $I \cap P_{L}=I_{L}$. Now $\left(A_{n}\right)_{q}$ is the image of $P_{L}$ under the quotient map, so $\left(A_{n}\right)_{q} \simeq P_{L} /\left(I \cap P_{L}\right)=P_{L} / I_{L}$. On the other hand, $I_{L}$ is contained in the kernel of the natural homomorphism of $P_{L}$ onto $A_{n}(q)$, so this homomorphism factors through $\left(A_{n}\right)_{q}$. This proves (a).

For (b), note first that the image of $F$ in $A_{n}(q)$ spans $A_{n}(q)$. We show that it is a basis. For each $i \quad(1 \leq i \leq n-1)$ and for each $w \in F$ there is a unique $w^{\prime}=w^{\prime}(i, w) \in F$ and $\alpha_{i w}^{w^{\prime}} \in \mathbf{C}[x]_{(x-q)}$ such that $e_{i} w=\alpha_{i w}^{w^{\prime}} w^{\prime}$. One can define a representation of $A_{n}(q)$ on the C-span of $F$ in $\left(A_{n}\right)_{q}$ by $e_{i}(q) w=\alpha_{i w}^{w^{\prime}}(q) w^{\prime}$. For $w \in F$, one checks that $w(q) 1=w$. Consequently the image of $F$ is linearly dependent.

To prove (c), take for each element of a basis of $e(q) A_{n}(q) f(q)$ a pre-image in $e\left(A_{n}\right)_{q} f$. If these elements were linearly dependent over $\mathbf{C}(x)$, then the linear dependence could be re-written with relatively prime coefficients in $\mathbf{C}[x]$, and evaluation at $q$ would then give a non-trivial linear dependence of the basis elements of $e(q) A_{n}(q) f(q)$. This gives the inequality $\operatorname{dim}_{\mathrm{C}} e(q) A_{n}(q) f(q) \leq \operatorname{dim}_{\mathrm{C}(x)} e A_{n} f$. Similar inequalities hold with $e$ replaced by $1-e$ and/or $f$ replaced by $1-f$. If any of these inequalities were strict, then adding them would give $\operatorname{dim}_{\mathrm{C}} A_{n}(q)<\operatorname{dim}_{\mathrm{C}(x)} A_{n}$, contradicting (b).

Another proof of $(\mathrm{b})$ consists in identifying $A_{n}(q)$ with the subalgebra of Brauer's centralizer algebra spanned by all diagrams with no intersecting edges (see $[\mathbf{B r}]$ and $[\mathbf{L}])$. One can also give a proof of (a) using this idea.

The following observation, although completely evident, is essential to our method of obtaining algebraic identities for $A_{n}(q)$ via matrix representations of $A_{n}$ which are not well defined for $A_{n}(q)$.

Evaluation principle. Any algebraic identity between evaluable etements in $A_{n}$ with coefficients in $\mathbf{C}[x]_{q}$ yields by evaluation an identity in $A_{n}(q)$.

0.3. Representations and path idempotents. We shall now describe irreducible representations for the Hecke algebra, following [W2], and 
identify those which factor through the Temperley-Lieb algebra. Recall that a Young (or Ferrers) diagram $\lambda=\left[\lambda_{1}, \lambda_{2}, \ldots, \lambda_{k}\right]$ is a left justified array of boxes with $\lambda_{i}$ boxes in the $i$ th row and $\lambda_{i} \geq \lambda_{i+1}$ for all $i$. For example,

$$
[5,3,1]=\bigoplus^{1}
$$

For $n \geq 0$ let $\Lambda_{n}$ denote the set of Young diagrams with $n$ boxes. Young's lattice is the graph whose vertices are labelled by Young diagrams, with two vertices connected by an edge if the corresponding diagrams differ by only one box. It is well known that the simple components of $\mathbf{C} S_{n}$ are labelled by the elements of $\Lambda_{n}$ and that the restriction to $S_{n-1}$ of the representation labelled by $\lambda \in \Lambda_{n}$ decomposes into the direct sum of the representations of $S_{n-1}$ labelled by all subdiagrams of $\lambda$ with $n-1$ boxes. Hence the Bratteli diagram of the $S_{n}$ 's is Young's lattice, with the elements of $\Lambda_{n}$ arranged on the $n$th level.

For $\lambda \in \Lambda_{n}$, a standard tableau $t$ of shape $\lambda$ is a filling of the diagram $\lambda$ with the numbers $1,2, \ldots, n$ so that the entries increase to the right in each row and downwards in each column. Let $T(\lambda)$ denote the set of standard tableaux of shape $\lambda$, and $T_{n}$ the set of all standard tableaux with $n$ boxes. Observe that $t \in T(\lambda)$ may be identified with a path on Young's lattice from [1] to $\lambda$, i.e. an increasing sequence of Young diagrams

$$
[1]=\lambda^{(1)} \subset \lambda^{(2)} \subset \cdots \subset \lambda^{(n)}=\lambda,
$$

where $\lambda^{(i)}$ is the diagram which consists of the boxes of $t$ containing the numbers $1,2, \ldots, i$. By a subtableau of $t$, we will always mean a standard subtableau,

$$
[1]=\lambda^{(1)} \subset \lambda^{(2)} \subset \cdots \subset \lambda^{(k)} \text { for some } k \leq n .
$$

We denote by $t^{\prime}$ the subtableau of size $n-1$.

For $t \in T_{n}$ and $1 \leq i \leq n-1$, define

$$
d(t, i)=c(i)-c(i+1)+r(i+1)-r(i),
$$

with $c(j)$ and $r(j)$ denoting column and row of the box containing the number $j$. Observe that $|d(t, i)|$ is the length of the shortest walk on the tableau $t$, with vertical and horizontal steps, between the cell containing $i$ and the cell containing $i+1$, and that the sign depends 
on whether $i+1$ is northeast or southwest of $i$ in $t$. Define for $d \in \mathbf{N} \backslash\{0\}$ the rational function

$$
a_{d}(x)=\frac{1-x^{d+1}}{(1+x)\left(1-x^{d}\right)}
$$

In [W2] and [H] an irreducible representation $\pi_{\lambda}$ of the Hecke algebra $H_{n}$ is defined on the vector space $V_{\lambda}$ with (orthonormal) basis labelled by $T(\lambda)$ in the following way. These representations are $q$-analogues of Young's normal representations. Let $\sigma_{i}(t)$ be the tableau obtained by interchanging the numbers $i$ and $i+1$ in the standard tableau $t$. Then define

$$
\pi_{\lambda}\left(e_{i}\right)=a_{d} t+\sqrt{a_{d} a_{-d}} \sigma_{i}(t),
$$

where $d=d(t, i)$. Observe that if $t$ is a standard tableau, $\sigma_{i}(t)$ fails to be a standard tableau only if $i$ and $i+1$ are in the same row or in the same column of $t$. But in this case, $a_{d} a_{-d}=0$, so the representation stays within standard tableaux. Note that $t \mapsto t^{\prime}$ induces a linear isomorphism between $V_{\lambda}$ and $\bigoplus_{\nu \subset \lambda, \nu \in \Lambda_{n-1}} V_{\nu}$, which is evidently an $H_{n-1}$-module isomorphism. Thus

$$
\pi_{\lambda \mid H_{n-1}} \cong \bigoplus_{\nu \subset \lambda, \nu \in \Lambda_{n-1}} \pi_{\nu}
$$

The proof of the irreducibility of the $\pi_{\lambda}$ is inductive: since the $\pi_{\nu}$ for $\nu \in \Lambda_{n-1}$ can be assumed irreducible, it suffices to observe that $V_{\nu} e_{n-1} V_{\mu} \neq 0$ if both $\nu$ and $\mu$ are obtained by removing one box from $\lambda$, and this follows easily from the definition of $\pi_{\lambda}$. One can also show inductively that the representations $\pi_{\lambda}$ for distinct $\lambda$ are mutually inequivalent. Since $\sum_{\lambda} \operatorname{dim}\left(\pi_{\lambda}\right)^{2}=n !=\operatorname{dim} H_{n}$, it follows that $H_{n}$ is semisimple. Similarly, if $q$ is not a root of unity, complex representations of $H_{n}(q)$ are defined by the same formulas, and they are likewise irreducible and mutually inequivalent, which shows that $H_{n}(q)$ is semisimple for such $q$. One can check directly that the representations $\pi_{\lambda}$ factor through the Temperley-Lieb algebra $A_{n}$ if $\lambda$ has no more than two rows. Since the sum of the squares of the dimensions of these is the Catalan number $C_{n}$, this is a complete family of inequivalent irreducible representations of $A_{n}$. Thus one has the Bratteli diagram shown in Figure 0.1 for the inclusions of the Temperley-Lieb algebras $A_{n}$ or $A_{n}(q)$ for $q$ not a root of unity.

Fix $\lambda \in \Lambda_{n}$ and let $z_{\lambda}$ denote the corresponding minimal central indempotent in $H_{n}$ defined by $\operatorname{ker}\left(\pi_{\lambda}\right)=\left(1-z_{\lambda}\right) H_{n}$. For each $t \in$ 


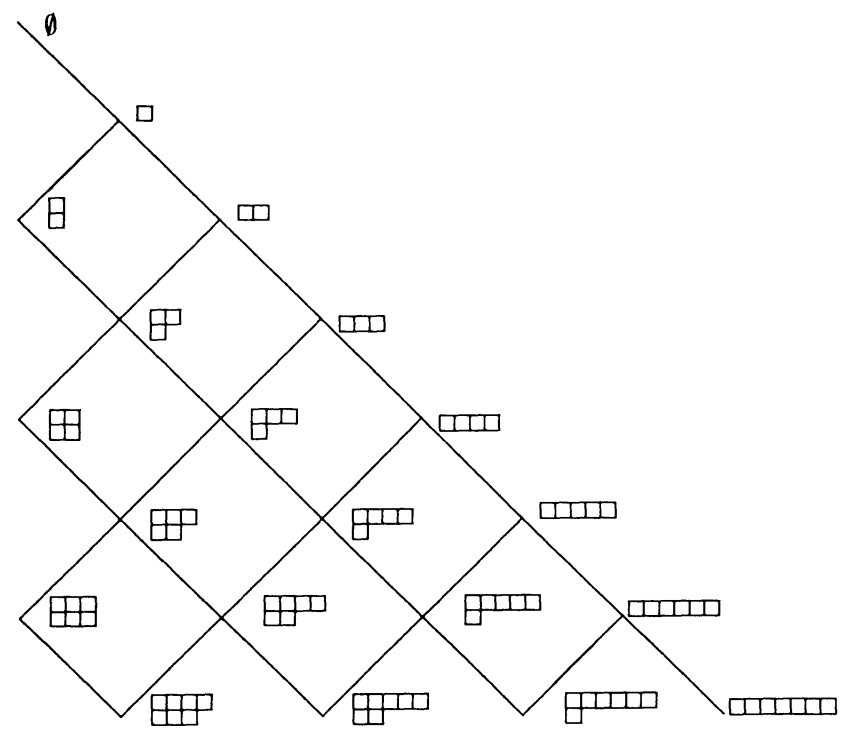

Figure 0.1. Bratteli diagram for $A_{n}$

$T(\lambda)$, let $p_{t}$ be the orthogonal projection of $V_{\lambda}$ onto $\mathbf{C} t$. Since $\pi_{\lambda}$ is an isomorphism of $z_{\lambda} H_{n}$ onto $\operatorname{End}\left(V_{\lambda}\right), p_{t}$ may also be regarded as an element of $z_{\lambda} H_{n}$. The idempotent $p_{t}$ can be evaluated inductively in the following way. Let $r \in T_{n-1}$. Using the definition of the representations $\pi_{\lambda}$ and the restriction rule (0.1), one obtains

$$
\pi_{\lambda}\left(p_{r} e_{n-1} p_{r}\right) t= \begin{cases}a_{d(t, n-1)} t & \text { if } t^{\prime}=r \\ 0 & \text { otherwise. }\end{cases}
$$

Since one can show that $a_{d(t, n-1)} \neq a_{d(s, n-1)}$ for any two extensions $t \neq s$ of $r$, one can compute the $p_{t}$ 's as spectral idempotents of $p_{r} e_{n-1} p_{r}$; namely if $r=t^{\prime}$, then

$$
p_{t}=\prod_{s} \frac{p_{r} e_{n-1} p_{r}-a_{d(s, n-1)} p_{r}}{a_{d(t, n-1)}-a_{d(s, n-1)}},
$$

where the product is over all $s \in T_{n}$ such that $s \neq t$ but $s^{\prime}=t^{\prime}=r$.

It is important to observe that for the Temperley-Lieb algebra one needs to take into consideration only tableaux belonging to diagrams with two rows at the most. In this case, there is at most one tableau $s$ such that $s \neq t$ but $s^{\prime}=t^{\prime}=r$, so one obtains path idempotents $p_{t}$ in the Temperley-Lieb algebra as follows:

$$
\begin{array}{ll}
p_{t}=p_{r} & \text { if } r \text { has only one extension, and } \\
p_{t}=\frac{p_{r} e_{n-1} p_{r}-a_{d(s, n-1)} p_{r}}{a_{d(t, n-1)}-a_{d(s, n-1)}} & \text { if } s^{\prime}=t^{\prime}=r .
\end{array}
$$


Furthermore, either $a_{d(t, n-1)}=0$ or $a_{d(s, n-1)}=0$, so the latter formula becomes

$$
\begin{array}{ll}
p_{t}=\left(a_{d(t, n-1)}\right)^{-1} p_{r} e_{n-1} p_{r} & \text { if } a_{d(t, n-1)} \neq 0, \\
p_{t}=p_{r}-\left(a_{d(s, n-1)}\right)^{-1} p_{r} e_{n-1} p_{r} & \text { otherwise. }
\end{array}
$$

It is evident that these formulas are evaluable in $A_{n}(q)$ if $q$ is not a root of unity. In the following section, we shall show that certain of the path idempotents are still evaluable when $q$ is a root of unity, and moreover, even when the individual path idempotents are not evaluable, certain sums of them are still evaluable.

1. Path idempotents and nilpotents. In this section and the next, $q$ will denote a fixed primitive $l$ th root of unity $(l \geq 3)$. We collect here various preliminary results needed for our analysis of the TemperleyLieb algebras $A_{n}(q)$. Recall that an element of the generic TemperleyLieb algebra $A_{n}$ is called evaluable if it is a linear combination of words in the generators $e_{i}$ with rational coefficients having no poles at $q$. Our method is to identify idempotents, matrix units, and nilpotents in $A_{n}(q)$ by analyzing evaluable elements in $A_{n}$.

With this in mind, we adopt the following convention: two evaluable idempotents $e, f$ in $A_{n}$ will be called equivalent only if there is an equivalence between them which is implemented by evaluable elements; that is, there exist evaluable $u, v$ in $A_{n}$ such that $e=u v$ and $f=v u$.

We will also need the following definitions:

Definitions. (a) A diagram $\lambda$ is called critical if $w(\lambda)=\lambda_{1}-\lambda_{2}+1$ is divisible by $l$. The $m$ th critical line on the Bratteli diagram for the generic Temperley-Lieb algebra is the line containing the diagrams $\{\lambda: w(\lambda)=m l\}$ (see Figure 1.1). A tableau $t$ of shape $\lambda$ is called critical if $\lambda$ is a critical diagram. We refer to the largest proper critical subtableau of $t$ (if any) simply as the critical subtableau of $t$.

(b) If a tableau $t$ has a proper critical subtableau, then the conjugate $\bar{t}$ of $t$ is defined to be the tableau obtained by leaving the critical subtableau $r$ of $t$ invariant and by reflecting $t \backslash r$ in the critical line containing the endpoint of $r$ (if this reflection exists); see Figure 1.2 on p. 318. For any tableau $t$, we define $p_{[t]}=p_{t}+p_{\bar{t}}$ if $\bar{t}$ exists; otherwise we set $p_{[t]}=p_{t}$.

(c) We say a critical tableau $t$ is evaluable if $p_{t}$ is evaluable. We say that a non-critical tableau is evaluable if $p_{[t]}$ is evaluable. 


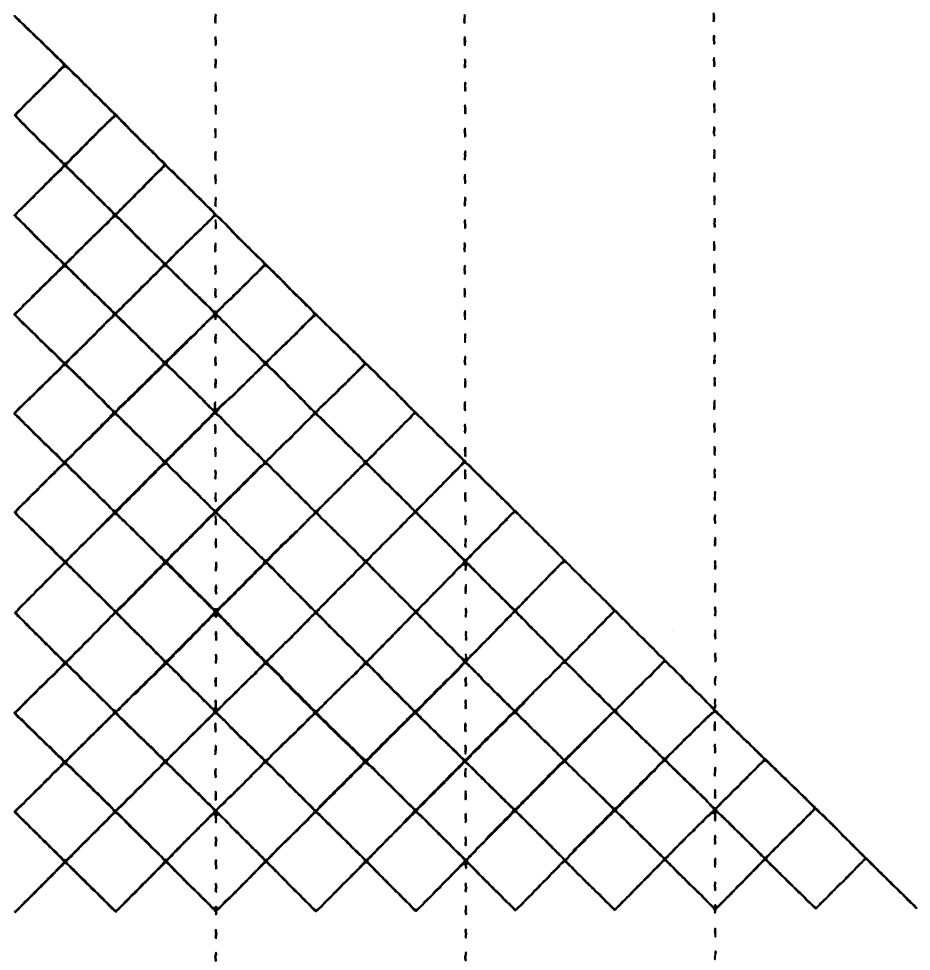

FIgURE 1.1. Critical lines

(d) A tableau $t$ is called regular if any two successive critical diagrams on $t$ are on different critical lines.

REMARKS. 1. We will see eventually that every regular tableau is evaluable.

2. There is one instance in which $t$ has a proper critical subtableau but $\bar{t}$ does not exist, namely if the endpoint of the critical subtableau is on the first critical line, and the endpoint of $t$ is on the second critical line.

LEMMA 1.1. Let $t=\left([1], \lambda^{(2)}, \ldots, \lambda^{(k)}=\lambda\right)$ be a tableau with no proper critical subtableau (i.e. such that $w\left(\lambda^{(i)}\right)<l$ for $\left.1 \leq i<k-1\right)$. Then $t$ is evaluable. Furthermore, if $t$ and $s$ are two such tableaux of the same shape, then $p_{t}$ is equivalent to $p_{s}$.

Proof. The proof, which goes by induction on $k$, is the same as in the generic case, and is contained in [W2]. The interpolation formula for $p_{t}$ from [W2] is $p_{t}=\left(a_{d}\right)^{-1} p_{t^{\prime}} e_{k-1} p_{t^{\prime}}$, or $p_{t}=p_{t^{\prime}}-$ $\left(a_{d}\right)^{-1} p_{t^{\prime}} e_{k-1} p_{t^{\prime}}$, where $d$ satisfies $|d| \leq l-2$ or $d=1-l$. In each 


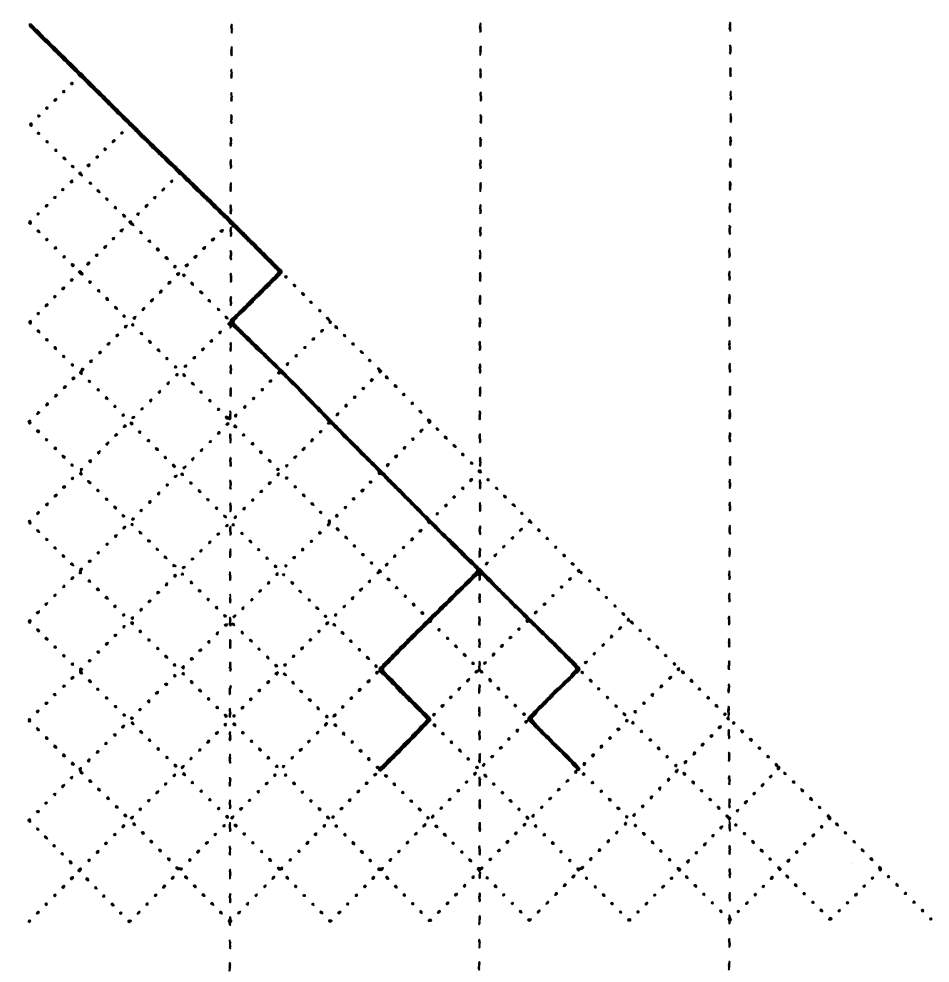

FIGURE 1.2. Conjugate tableaux

case, $a_{d}$ is non-zero and non-singular at $q$, so if $p_{t^{\prime}}$ is evaluable at $q$, then so is $p_{t}$. If $t$ and $s$ are two such tableaux of shape $\lambda$, then one can be obtained from the other by applying a sequence of adjacent transpositions $\sigma_{i}$, with $2 \leq i \leq k-1$. Therefore it suffices to consider the case that $s=\sigma_{i}(t)$ for some such $i$. The equivalence is then implemented by $u=1 /\left(a_{d} a_{-d}\right) p_{t} e_{i} p_{s}, v=p_{s} e_{i} p_{t}$, with $d=d(t, i)$.

LEMMA 1.2 (Little Diamond Lemma). Let $r$ be an evaluable critical tableau of shape $\mu$ and length $k$. Set $\lambda=\mu+[1,1]$. Let $s$ denote the unique tableau of length $k+2$ extending $r$ and ending at $\lambda$ for which $t=\sigma_{k}(s)$ is standard. (See Figure 1.3.) Then there exists a 3-by-3 system of evaluable matrix units in $A_{k+2}$ with the diagonal units equal to $p_{t}, p_{r} e_{k+1}$ and $p_{r}\left(1-e_{k+1}\right) z_{\lambda}$. In particular $p_{r} z_{\lambda}$ is evaluable.

Proof. The three dimensional subspace $V$ of $V_{\lambda}$ spanned by $s, \bar{s}$, and $t$ is invariant under the action of $e_{k}, e_{k+1}$ and $p_{r}$. Set $u=$ $\left(1-p_{r}\right) e_{k} e_{k+1} p_{r}$ and $u^{*}=e_{k+1} p_{r} e_{k}\left(1-p_{r}\right)$. Computation with the 


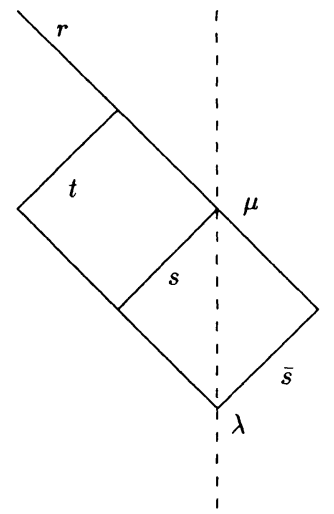

Figure 1.3. Little Diamond Lemma

matrix representatives on $V$ show that

$$
\begin{aligned}
p_{t} & =\frac{1}{a_{ \pm m l} a_{m l \pm 1} a_{-(m l \pm 1)}} u u^{*}, \quad \text { while } \\
p_{r} e_{k+1} & =\frac{1}{a_{ \pm m l} a_{m l \pm 1} a_{-(m l \pm 1)}} u^{*} u .
\end{aligned}
$$

Since $a_{ \pm m l}$ has a simple pole at $x=q$ while the product $a_{m l \pm 1} a_{-(m l \pm 1)}$ has a simple zero, it follows that $p_{t}$ is evaluable and equivalent to $p_{r} e_{k+1}$.

A similar computation shows that $p_{r}\left(1-e_{k+1}\right) z_{\lambda}$ is evaluable and equivalent to $p_{t}$. Finally, the sum $p_{r} z_{\lambda}=p_{r} e_{k+1}+p_{r} z_{\lambda}\left(1-e_{k+1}\right)$ is evaluable.

LEMMA 1.3 (Interpolation Lemma). Let $t$ be a tableau with evaluable critical subtableau $r$. Then:

(a) $p_{[t]}$ is evaluable. In particular, if $r$ ends on the first critical line and $t$ ends on the second critical line, then $p_{t}$ is evaluable.

(b) Let $s$ be another tableau with the same critical subtableau $r$ and the same shape as $t$. Then $p_{[s]}$ and $p_{[t]}$ are equivalent.

Proof. (a) Write $t=\left([1], \lambda^{(2)}, \ldots, \lambda^{(n)}\right)$, and let $k$ denote the length of the critical subtableau $r$. Suppose that $r$ ends on the $m$ th critical line at the diagram $\mu$. Also, suppose without loss of generality that $\lambda^{(n)}$ is weakly to the right of the $m$ th critical line; that is, $w\left(\lambda^{(n)}\right) \geq m l$.

We proceed by induction on $n-k$. If $n-k=1$, then $p_{[t]}=p_{r}$, which is evaluable by hypothesis. If $n-k=2$, then $p_{[t]}=p_{r} z_{(\mu+[1,1])}$ 
or $p_{[t]}=p_{r}\left(1-z_{(\mu+[1,1])}\right)$; in either case, $p_{[t]}$ is evaluable by Lemma 1.2. Suppose now that $n-k \geq 3$ and that $p_{\left[t^{\prime}\right]}$ is evaluable.

Case 1. $\lambda^{(n)}=\lambda^{(n-2)}+[1,1]$.

Set $d=d(t, n-1)$ and $\bar{d}=d(\bar{t}, n-1)$. Then $a_{d}$ and $a_{\bar{d}}$ are nonzero and non-singular at $q$, and $a_{d}(q)=a_{\bar{d}}(q)$. Let $s$ be the unique tableau such that $s \neq t$ and $s^{\prime}=t^{\prime}$, and consider the space $V$ with basis $\{\bar{s}, \bar{t}, t, s\}$. (If $m=1$ and $s$ ends on the second critical line, then $\bar{s}$ does not exit; in this case take instead $V=\operatorname{span}\{\bar{t}, t, s\}$.) On $V$, the element $E=p_{\left[t^{\prime}\right]} e_{n-1} p_{\left[t^{\prime}\right]}$ has matrix $\operatorname{diag}\left(0, a_{\bar{d}}, a_{d}, 0\right)$ (resp. $\operatorname{diag}\left(a_{\bar{d}}, a_{d}, 0\right)$ in the special case). Moreover, observe that $p_{[t]}$ is given by the matrix $\operatorname{diag}(0,1,1,0)$ (resp. $\operatorname{diag}(1,1,0))$. Hence it follows that

$$
p_{[t]}=\left(a_{d} a_{\bar{d}}\right)^{-1}\left(\left(a_{d}+a_{\bar{d}}\right) E-E^{2}\right),
$$

so $p_{[t]}$ is evaluable.

Case 2. $\lambda^{(n)}=\lambda^{(n-2)}+[2,0]$ or $\lambda(n)=\lambda^{(n-2)}+[0,2]$.

With $s$ defined as above, $p_{[s]}$ is evaluable by Case 1 , and thus $p_{[t]}=p_{\left[t^{\prime}\right]}-p_{[s]}$ is evaluable. In the special case that $m=1$ and $t$ ends on the second critical line, observe that $\bar{t}$ is not defined but $p_{t}=p_{\left[t^{\prime}\right]}-p_{[s]}$, so $p_{t}$ is evaluable.

(b) Our assumptions imply that $\bar{s}$ exists if and only if $\bar{t}$ exists; suppose this is so, the other case being similar. Now the proof is essentially the same as that of the equivalence statement in Lemma 1.1 , except that we will have to adjust our initial choice $u, u^{*}$ for elements implementing the equivalence because the representing matrices for $u^{*} u$ and $u u^{*}$ have two distinct non-zero eigenvalues.

The tableau $s$ can be obtained from $t$ by applying a sequence of adjacent transpositions. Therefore it suffices to suppose that $s=\sigma_{i}(t)$ for some $i$. Set $u^{*}=p_{[t]} e_{i} p_{[s]}$ and $u=p_{[s]} e_{i} p_{[t]}$. On the space with basis $\{\bar{s}, \bar{t}, t, s\}$, the element $u^{*} u$ has matrix $\operatorname{diag}\left(0, c_{-}, c_{+}, 0\right)$ and $u u^{*}$ has matrix $\operatorname{diag}\left(c_{-}, 0,0, c_{+}\right)$, where the rational functions $c_{-}$ and $c_{+}$are non-zero and non-singular $q$. It follows that

$$
\left(c_{-} c_{+}\right)^{-1}\left(u^{*} u-c_{-}\right)\left(u^{*} u-c_{+}\right)\left(p_{[s]}+p_{[t]}\right)=p_{[s]} .
$$

Expanding this and solving for $p_{[t]}$ gives

$$
p_{[t]}=u^{*} v \text { and } p_{[s]}=v u^{*},
$$

where $v=-\left(c_{-} c_{+}\right)^{-1}\left(u u^{*} u-\left(c_{-}+c_{+}\right) u\right)$. 


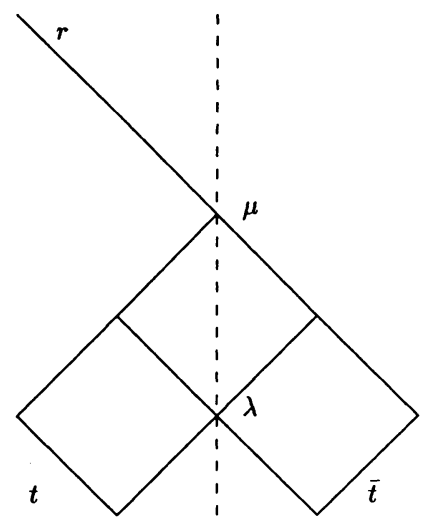

FIGURE 1.4

COROLlaRy 1.4. Let $r$ be an evaluable critical tableau of length $k$ and shape $\mu$. Set $\lambda=\mu+[1,1]$. Consider the six extensions of $r$ of length $k+3$ which end at $\mu+[2,1]$ or at $\mu+[1,2]$, and let $t$ denote the leftmost of these extensions in Figure 1.4. Then there is a 3-by-3 system of evaluable matrix units in $A_{k+3}$ with diagonal matrix units $p_{[t]}, p_{r} e_{k+1}$, and $p_{r}\left(1-e_{k+1}\right) z_{\lambda}$.

Proof. Observe that $p_{r} z_{\lambda}$ and $p_{[t]}$ are evaluable by Lemmas 1.2 and 1.3. One obtains the equivalences by the same computations as in Lemma 1.2, where one has to add a correction term for the matrix units as in Lemma 1.3(b).

The following lemma concerns a rational function which appears as the trace of a certain evaluable essential idempotent in the TemperleyLieb algebra over $\mathbf{C}(x)$. We need to show that this quantity is nonzero and non-singular at $x=q$ in order to know that the evluation of the algebra element at $x=q$ is still an essential idempotent.

LEMMA 1.5. Fix $m \geq 1$. The function

$$
T_{k}=\left(a_{m l-1} a_{1-m l} a_{-m l}\right)^{k-1} a_{-m l}+\left(a_{m l+1} a_{-(m l+1)} a_{m l}\right)^{k-1} a_{m l}
$$

has a removable singularity at $q$, and

$$
T_{k}(q)=\tau^{k}\left(q+4-2 k+q^{-1}\right),
$$

where $\tau=q /(1+q)^{2}$. In particular $T_{k}(q) \neq 0$ for $k \geq 3$.

Proof. It suffices to prove this for $m=1$ since the $l$ th root of unity $q$ is in particular an $m l$ th root of unity. Let $t_{k}$ denote the 
quantity $\tau^{k}\left(q+4-2 k+q^{-1}\right)$. We find a recursion for $T_{k}$ and for $t_{k}$. First $T_{1}=a_{l}+a_{-l}=1=t_{1}$. Denote $D_{-}=a_{l-1} a_{1-l} a_{-l}$ and $D_{+}=a_{l+1} a_{-(l+1)} a_{l}$. Then $D_{-}$and $D_{+}$are non-singular at $q$ with value $D_{-}(q)=D_{+}(q)=\tau$. We have

$$
\begin{aligned}
T_{k+1} & =D_{-}^{k} a_{-l}+D_{+}^{k} a_{l} \\
& =D_{-}\left(D_{-}^{k-1} a_{-l}+D_{+}^{k-1} a_{l}\right)+D_{+}^{k-1}\left(D_{+}-D_{-}\right) a_{l} \\
& =D_{-} T_{k}+D_{+}^{k-1}\left(D_{+}-D_{-}\right) a_{l} .
\end{aligned}
$$

Now one computes that

$$
\left(D_{+}-D_{-}\right) a_{l}(x)=\frac{x^{2}\left(1+x^{l}\right)(1-x)}{(1+x)^{4}\left(x-x^{l}\right)}
$$

so that $\left(D_{+}-D_{-}\right) a_{l}(q)=-2 \tau^{2}$. Assuming inductively that $T_{k}$ is non-singular at $q$ and that $T_{k}(q)=t_{k}$, we obtain that $T_{k+1}$ is nonsingular as well and

$$
T_{k+1}(q)=\tau t_{k}-2 \tau^{k+1}=t_{k+1} .
$$

Because $0 \leq\left|q+q^{-1}\right|<2$, the quantity $t_{k}$ is non-zero for all $k \geq 3$.

LEMMA 1.6 (Big Diamond Lemma). Let $t$ be a tableau such that both $t$ and its critical subtableau $r$ end on the same critical line. Suppose that $p_{r}$ is evaluable. Then $p_{[t]}$ dominates an evaluable minimal idempotent $f$ whose matrix coefficients on the space with basis $\{\bar{t}, t\}$ all have simple poles $x=q$.

Proof. Suppose that $r$ ends at the diagram $\mu$ of size $k$ on the $m$ th critical line and that $t$ ends at $\mu+[n, n]$. We consider first instead of the original $t$ the following zig-zag path $s$ (see Figure 1.5):

$$
s=\left(r, \mu^{(k+1)}, \ldots, \mu^{(k+2 n)}=\lambda+[n, n]\right),
$$

where

$$
\begin{aligned}
\mu^{(k+2 j)} & =\mu+[j+1, j-1] & & \text { for } 1 \leq j \leq n-1, \\
\mu^{(k+2 j+1)} & =\mu+[j+1, j] & & \text { for } 0 \leq j \leq n-1 .
\end{aligned}
$$

We show that $p_{[s]}$ dominates an evaluable minimal idempotent $p$ whose matrix coefficients on the space spanned by $\{s, \bar{s}\}$ have simple poles at $x=q$. Define

$$
w=\left(e_{k+1} e_{k+3} \cdots e_{k+2 n-1}\right)\left(e_{k+2} e_{k+4} \cdots e_{k+2 n-2}\right) p_{[s]}
$$




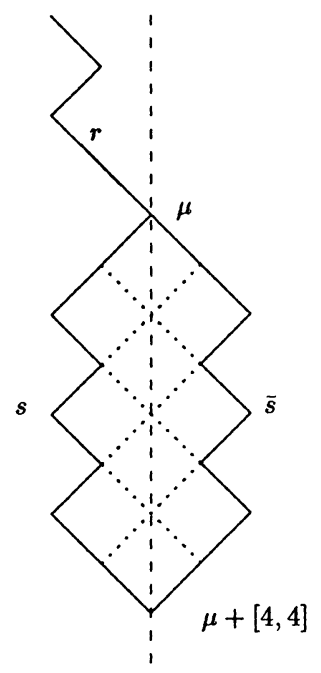

Figure 1.5. Big Diamond Lemma

and

$$
w^{*}=p_{[s]}\left(e_{k+2} e_{k+4} \cdots e_{k+2 n-2}\right)\left(e_{k+1} e_{k+3} \cdots e_{k+2 n-1}\right) .
$$

The matrix of $w^{*} w$ on the space with basis $\{\bar{s}, s\}$ is

$$
\left[\begin{array}{cc}
\left(a_{m l-1} a_{1-m l} a_{-m l}\right)^{n-1} a_{-m l} & \sqrt{ } \\
\sqrt{ } & \left(a_{m l+1} a_{-(m l+1)} a_{m l}\right)^{n-1} a_{m l}
\end{array}\right],
$$

where the off-diagonal entries are the square root of the product of the diagonal entries. The trace of this matrix is the rational function $T_{n}$ of Lemma 1.5, which is non-zero and non-singular at $q$. Therefore $p:=T_{n}^{-1} w^{*} w$ is a minimal idempotent with the desired properties. Rewrite the matrix of $p$ as $\left[\begin{array}{ll}b & \sqrt{ } \\ \sqrt{ } & 1-b\end{array}\right]$.

It remains to observe that the equivalence between $p_{[s]}$ and $p_{[t]}$ which is given by Lemma $1.3(\mathrm{~b})$ preserves th $\geq$ poles of the matrix representatives. For this, note that the matrices of the implementing elements $u, u^{*}$ on the space spanned by $\{\bar{t}, \bar{s}, s, t\}$ are of the form

$$
u\left[\begin{array}{cccc}
0 & \alpha_{-} & & \\
0 & 0 & & \\
& & 0 & 0 \\
& & \alpha_{+} & 0
\end{array}\right] \text { and } u^{*}=\left[\begin{array}{cccc}
0 & 0 & & \\
\alpha_{-}^{-1} & 0 & & \\
& & 0 & \alpha_{+}^{-1} \\
& & 0 & 0
\end{array}\right]
$$

where $\alpha_{ \pm}$are rational functions which are non-zero and non-singular at $q$. A simple matrix computation shows that $f=u p u^{*}$ has the desired properties.

Several of our lemmas have hypothesized an evaluable critical tableau, but so far the only such tableaux which we have in hand are 
those provided by Lemmas 1.1 and 1.3(a). We are now in the position to produce a sufficient family of evaluable critical tableaux of any critical shape. Recall that a tableau is called regular if its successive critical diagrams lie on different critical lines.

LEMMA 1.7. (a) Every regular critical tableau is evaluable.

(b) If $s$ and $t$ are regular critical tableaux with the same critical subtableau and the same shape, then $p_{s}$ and $p_{t}$ are equivalent.

Proof. The proof of (a) is by induction on the number of critical diagrams on the tableau $t$. If this number is one or two, the conclusion follows from Lemmas 1.1 and 1.3(a). So suppose that $t$ has at least three critical diagrams and that every regular critical tableau with fewer critical diagrams is evaluable.

Consider the case that $t=\left([1], \lambda^{(2)}, \ldots, \lambda^{(k)}=\lambda\right)$ has its last three critical diagrams $\lambda^{(i)}, \lambda^{(j)}$, and $\lambda^{(k)}=\lambda$ on the $m$ th,$(m \pm 1)$ st, and $m$ th critical lines. We can assume that $\bar{t}$ exists, since otherwise the desired conclusion follows from the induction hypothesis and Lemma 1.3(a). Set $s=\sigma_{j}(t)$. See Figure 1.6. By the induction hypothesis and Lemma 1.3 , both $p_{[s]}$ and $p_{[t]}$ are evaluable and by Lemma 1.6, $p_{[s]}$ dominates an evaluable minimal idempotent $f$ whose matrix coefficients on the space with basis $\{s, \bar{s}\}$ have simple poles at $x=q$.

Let $b$ denote the matrix coefficient, $p_{s} f p_{s}=b p_{s}$. Define $u=$ $f e_{j} p_{[t]}$ and $u^{*}=p_{[t]} e_{j} f$. Computation with the matrix representatives of $f, e_{j}$, and $p_{[t]}$ on the space with basis $\{\bar{s}, s, t, t\}$ shows that

$p_{t}=\frac{1}{b a_{(m \pm 1) l \mp 1} a_{-((m \pm 1) l \mp 1)}} u^{*} u \quad$ and $\quad f=\frac{1}{b a_{(m \pm 1) l \mp 1} a_{-((m \pm 1) l \mp 1)}} u u^{*}$.

Since $b$ has a simple pole at $x=q$ while the product $a_{(m \pm 1) l \mp 1} \times$ $a_{-((m \pm 1) l \mp 1)}$ has a simple zero, it follows that $p_{t}$ is evaluable and equivalent to $f$.

If the last three critical diagrams on $t$ are all on different critical lines, then $\bar{t}$ is evaluable by the previous case, while $p_{[t]}$ is evaluable by the induction hypothesis and Lemma 1.3 , so $p_{t}$ is also evaluable.

For part (b), note that $s$ can be obtained from $t$ by applying a sequence of adjacent transpositions; the same proof as in Lemma 1.1 shows that $p_{s}$ and $p_{t}$ are equivalent.

LEMMA 1.8. Fix $m \geq 1$ and let $t$ be the unique tableau of shape $\lambda=[(m+1) l-1, l]$ which passes through the diagram $[(m+1) l-1]$. 


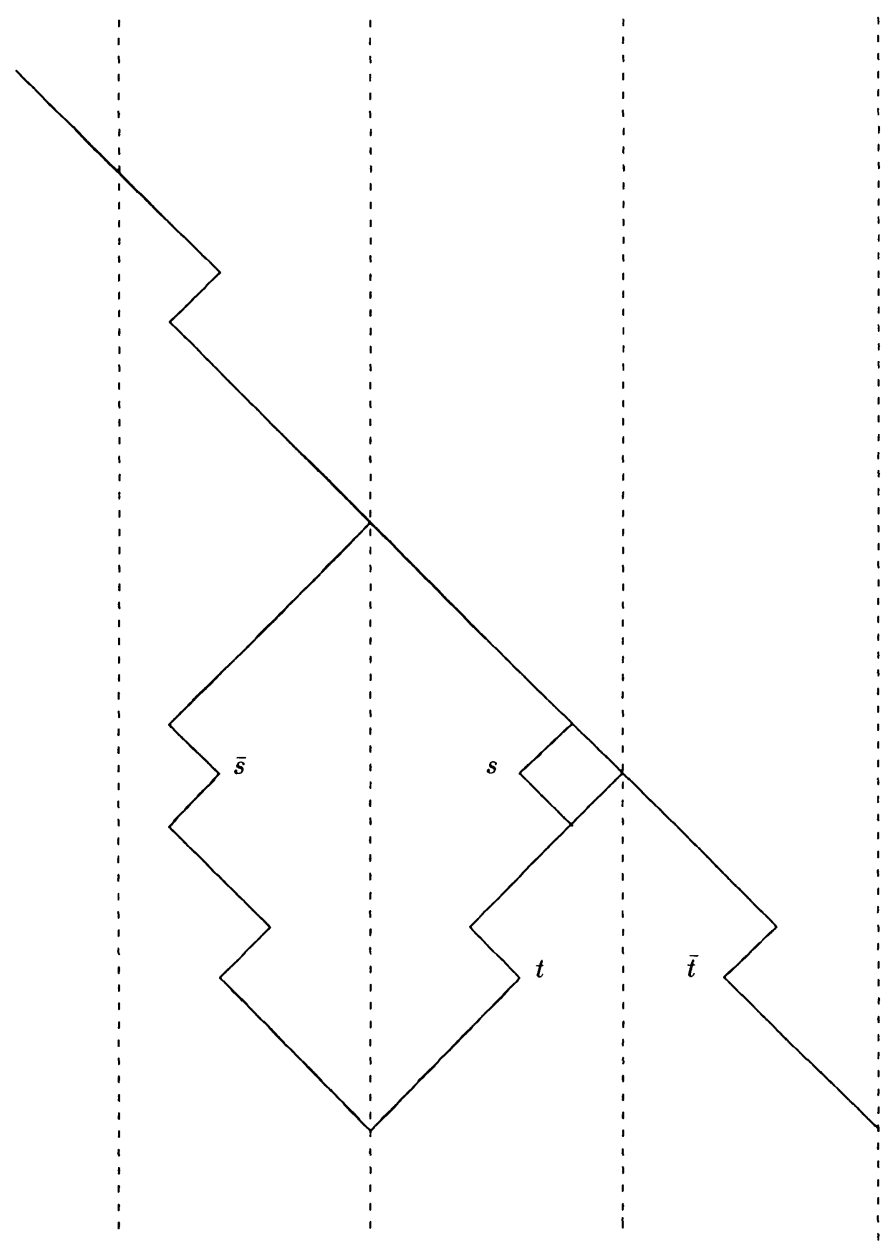

Figure 1.6

Then $p_{t}$ is equivalent to $p_{r}$ where $r$ is a tableau of shape $\lambda$ with critical subtableau of shape $[(m-1) l-1]$.

Proof. Set $s=\sigma_{(m+1) l-1}(t)$ and $r=\sigma_{m l-1}(\bar{s})$. Both $p_{t}$ and $p_{r}$ are evaluable by Lemma 1.7. Moreover, by the argument of Lemma 1.7, both are equivalent to $f$, where $f$ is the evaluable minimal idempotent dominated by $p_{[s]}$ which is given by Lemma 1.6.

Let $t$ be a non-critical tableau of length $n$ with a critical tableau $r$ of length $k$. In the generic algebra, the element $p_{[t]} e_{k} p_{[t]}$ is an essential idempotent, in fact essentially $p_{t}$ or $p_{\bar{t}}$. However, the evaluation of such elements in $A_{n}(q)$ gives non-zero nilpotents of order 2 which moreover are contained in the radical of $A_{n}(q)$. 
LEMMA 1.9 (Nilpotent elements). Let $t$ be a non-critical tableau with critical subtableau of shape $[m l-1]$ for some $m \geq 1$. Assume that the endpoint of $t$ is to the left of the $m$ th critical line. Write $e=e_{m l-1}$, $s=\sigma_{m l-1}(t)$, and $n_{[t]}=p_{[t]} e p_{[t]}$.

(a) $n_{[t]}(q) \neq 0$ but $n_{[t]}(q)^{2}=0$.

(b) $p_{[t]} A_{n} p_{[t]}(q)$ is two dimensional, isomorphic to $\mathbf{C}[x] /\left(x^{2}\right)$.

(c) $p_{[t]} A_{n} p_{[s]}(q)$ and $p_{[s]} A_{n} p_{[t]}(q)$ are one dimensional.

(d) $p_{[t]} A_{n} p_{[s]} A_{n} p_{[t]}(q)=\mathrm{Cn}_{[t]}(q)$ and

$$
p_{[s]} A_{n} p_{[t]} A_{n} p_{[s]}(q)= \begin{cases}(0) & \text { if } m=1 \\ \mathrm{Cn}_{[s]}(q) & \text { otherwise. }\end{cases}
$$

(e) $n_{[t]} p_{[t]} A_{n} p_{[s]}(q)=p_{[s]} A_{n} p_{[t]} n_{[t]}(q)=(0)$. If $m>1$, then

$$
p_{[t]} A_{n} p_{[s]} n_{[s]}(q)=n_{[s]} p_{[s]} A_{n} p_{[t]}(q)=(0) .
$$

Proof. The idempotents $p_{[t]}$ and $p_{[s]}$ are evaluable by Lemmas 1.7 and 1.3. For part (a), note that $p_{[t]} e p_{[t]}=p_{t} e p_{t}=a_{m l-1} p_{t}$, so $\left(p_{[t]} e p_{[t]}\right)^{2}=a_{m l-1} p_{[t]} e p_{[t]}$. This last relation can be evaluated at $q$ and gives $\left(p_{[t]} e_{k} p_{[t]}\right)^{2}(q)=0$. To see that $p_{[t]} e p_{[t]}(q) \neq 0$, let $T$ be an extension of $t$ ending on the $m$ th critical line and also having critical subtableau of shape $[m l-1]$. Let $f$ be an evaluable minimal idempotent dominated by $p_{[T]}$ whose matrix coefficients with respect to the basis $\{T, \bar{T}\}$ have simple poles at $x=q$; let $b$ denote the matrix coefficient, $p_{T} f p_{T}=b p_{T}$. Note that $f\left(p_{[t]} e p_{[t]}\right) f=a_{m l-1} f p_{t} f=$ $a_{m l-1} f p_{T} f=a_{m l-1} b f$. Since $a_{m l-1} b$ is neither zero nor singular at $q, f\left(p_{[t]} e_{k} p_{[t]}\right) f(q)=a_{m l-1} b f(q) \neq 0$.

(b) It follows from constancy of dimension that $p_{[t]} A_{n} p_{[t]}(q)$ is two dimensional, so that $\left\{p_{[t]}, n_{[t]}\right\}$ is a basis.

(c) Follows from constancy of dimension.

(d) Set $u=p_{[s]} e p_{[t]}$ and $u^{*}=p_{[t]} e p_{[s]}$. By the calculation of part (a), we have $a_{m l-1} p_{t}=n_{[t]}$, so that $u^{*} u=p_{t} e p_{s} e p_{t}=a_{m l-1} a_{1-m l} p_{t}=$ $a_{1-m l} n_{[t]}$. Now evaluating at $x=q$ gives $u^{*} u(q)=n_{[t]}(q)$. In particular, $u(q)$ and $u^{*}(q)$ are non-zero. If $m=1$, then $p_{s}$ is evaluable, and $u u^{*}=p_{s} e p_{t} e p_{s}=a_{m l-1} a_{2-m l} p_{s}$. Evaluating this at $x=q$ gives $u u^{*}(q)=0$. If $m>1$, then $u u^{*}=a_{m l-1} a_{1-m l} p_{s}=$ $a_{1-m l} a_{m l-1}\left(a_{(m-1) l-1}\right)^{-1} n_{[s]}$. Evaluating this at $x=q$ gives $u u^{*}(q)=$ $n_{[s]}(q)$.

(e) It follows from calculations similar to those in part (d) that $n_{[t]} u^{*}(q)=u n_{[t]}(q)=0$, and, if $m>1$, then also $u^{*} n_{[s]}(q)=$ $n_{[s]} u(q)=0$. 
2. The structure of $A_{n}(q)$. In this section we will complete the analysis of the structure of the Temperley-Lieb algebras $A_{n}(q)$ at a primitive $l$ th root of unity $q(l \geq 3)$. The first step is to identify idempotents which map to the minimal central idempotents in the maximal semisimple quotient of $A_{n}(q)$, and to analyze the reduction of $A_{n}(q)$ by these idempotents.

Let $\lambda$ be a diagram between the $m$ th and $m+1$ st critical line. Partition the set $T(\lambda)$ of tableaux of shape $\lambda$ into the classes $L(\lambda)$ and $R(\lambda)$, which consist of those tableaux $t$ whose critical subtableau ends in the $m$ th (for $L(\lambda)$ ), or in the $m+1$ st critical line (for $R(\lambda)$ ). If $m=0$, then $L(\lambda)$ consists of those tableaux of shape $\lambda$ having no intersection with a critical line. Let $f_{\lambda}, f_{\lambda}^{R}$, and $f_{\lambda}^{L}$ denote the cardinalities of $T(\lambda), R(\lambda)$, and $L(\lambda)$ respectively. Define

$$
z_{\lambda}^{R}=\sum\left\{p_{[t]}: t \in R(\lambda)\right\} \quad \text { and } \quad z_{\lambda}^{L}=\sum\left\{p_{[t]}: t \in L(\lambda)\right\} .
$$

The summands in these expressions are not necessarily evaluable. $\mathrm{Ob}$ serve that if $\mu$ is the image of $\lambda$ under the reflection in the $m+1$ st critical line, we have $z_{\lambda}^{R}=z_{\mu}^{L}$ and $f_{\lambda}^{R}=f_{\mu}^{L}$. The summation formula for $f_{\lambda}^{L}$ given in the introduction can be obtained as follows: $f_{\lambda}^{L}=f_{\lambda}-f_{\lambda}^{R}=f_{\lambda}-f_{\mu}^{L}$; iterating this step gives the formula $f_{\lambda}^{L}=f_{\lambda}-f_{\mu}+\cdots$.

We are going to show that

$$
\left\{z_{\lambda}^{L}(q): \lambda \text { non-critical and }|\lambda|=n\right\} \cup\left\{z_{\lambda}(q): \lambda \text { critical and }|\lambda|=n\right\}
$$

are all the minimal central idempotents of $A_{n}(q)$ modulo its radical and that the corresponding simple components are isomorphic to $f_{\lambda}^{L} \times$ $f_{\lambda}^{L}$ (resp. $f_{\lambda} \times f_{\lambda}$ ) matrix rings over $\mathbf{C}$.

We shall need some more notation for the inductive proof of this statement. Observe that any diagram $\lambda$ with two rows has at most two subdiagrams with one box less. We use the notation $\alpha=\lambda-[1,0]$ (the left subdiagram) and $\beta=\lambda-[0,1]$ (the right subdiagram), whenever these diagrams exist. Obviously, we have the identities

$$
f_{\lambda}=f_{\alpha}+f_{\beta}
$$

and, if both $\alpha$ and $\beta$ are noncritical,

$$
f_{\lambda}^{L}=f_{\alpha}^{L}+b_{\beta}^{L}, \quad f_{\lambda}^{R}=f_{\alpha}^{R}+f_{\beta}^{R} .
$$

If $\alpha$ is critical, then

$$
f_{\lambda}^{L}=f_{\alpha}+f_{\beta}^{L} \quad \text { and } \quad f_{\lambda}^{R}=f_{\beta}^{R},
$$

and similarly if $\beta$ is critical. 
In the following proposition we will construct a semisimple subalgebra of $A_{n}(q)$ which will turn out to be an isomorphic lifting of the maximum semisimple quotient of $A_{n}(q)$. We do not work directly in $A_{n}(q)$ but rather with evaluable elements of $A_{n}$. To construct the semisimple subalgebra, we assume inductively that we have a partition of unity of mutually orthogonal evaluable idempotents of $A_{n-1}$ and sufficiently many evaluable matrix units connecting them. Furthermore, we assume that each of the equivalence classes of idempotents contains an evaluable path idempotent $p_{t}$ or $p_{[t]}$ corresponding to a regular path $t$. Then by "refining" these path idempotents and applying the lemmas of the previous section, we obtain a partition of unity in $A_{n}$ with the same properties. Thus the inductive construction of the semisimple lifting is analogous to the proof of irreducibility of the representations $\pi_{\lambda}$ which we sketched in $\S 0.3$.

We recall our convention that equivalence of evaluable idempotents in $A_{n}$ means equivalence implemented by evaluable elements.

Proposition 2.1. (a) If $\lambda$ is a critical diagram, then $z_{\lambda}$ has a partition of unity consisting of $f_{\lambda}$ mutually orthogonal, mutually equivalent evaluable idempotents, among them at least one $p_{t}$ such that $t$ is regular of shape $\lambda$. Moreover, for any two regular tableaux $s, t$ of shape $\lambda, p_{s}$ is equivalent to $p_{t}$.

(b) If $\lambda$ is non-critical, then $z_{\lambda}^{L}$ has a partition of unity consisting of $f_{\lambda}^{L}$ mutually orthogonal, mutually equivalent evaluable idempotents, among them at least one $p_{[t]}$ for a regular $t \in L(\lambda)$. Moreover, if $s$ and $t$ are two such tableaux, then $p_{[s]}$ and $p_{[t]}$ are equivalent.

Proof. The proof is by induction on the size of the diagram $\lambda$. The statements hold for all diagrams with one row by Lemmas 1.7(a) and 1.3(a), and for all diagrams to the left of the first critical line by Lemma 1.1. We assume that they hold for all diagrams of size less then $|\lambda|$, that $\lambda$ has a non-zero second part, and that $\lambda$ is on or to the right of the first critical line. It suffices to produce the required number of mutually orthogonal, mutually equivalent evaluable idempotents dominated by $z_{\lambda}$ (resp. $z_{\lambda}^{L}$ ), including those of the specified types, since the sum of these is then necessarily equal to $z_{\lambda}$ (resp. $z_{\lambda}^{L}$ ).

First consider a diagram $\lambda$ on the $m$ th critical line. Set $\mu=$ $\lambda-[1,1]$, and let $r$ and $t$ be tableaux of shape $\mu$ and $\lambda$ respectively whose critical subtableaux are of shape $[(m-1) l-1]$ (or, if $m=1$, which have no proper critical subtableaux). These are regular, 
so evaluable by Lemma 1.1 or 1.7 . By the induction assumption and Lemma 1.1 or Lemma $1.3(\mathrm{~b}), z_{\alpha}^{L}$ has a partition of unity consisting of $f_{\alpha}^{L}$ mutually orthogonal, mutually equivalent evaluable idempotents in $A_{n-1}$, including $p_{\left[t^{\prime}\right]}$. From this we obtain the same number of mutually orthogonal, mutually equivalent idempotents in $A_{n}$, dominated by $z_{\alpha}^{L} z_{\lambda}$, and including $p_{t}$; namely, if $\left\{u_{i j}\right\}$ is a system of evaluable matrix units with $u_{11}=p_{\left[t^{\prime}\right]}$, then $\left\{v_{i j}=u_{i 1} p_{t} u_{1 j}\right\}$ is such a system with $v_{11}=p_{t}$. By Lemmas $1.7(\mathrm{~b})$ and 1.2, $p_{t}$ is equivalent to both $p_{r} e_{n-1}$ and $p_{r}\left(1-e_{n-1}\right) z_{\lambda}$. Using this and the induction hypothesis for $z_{\mu}$, we obtain $2 f_{\mu}=2 f_{\alpha}^{R}$ mutually orthogonal, mutually equivalent evaluable idempotents in $A_{n}$ dominated by $z_{\alpha}^{R} z_{\lambda}$, and all equivalent to $p_{t}$. If $\lambda_{2}<l$, this suffices, since $f_{\beta}^{R}=0$. Now suppose $\lambda_{2}=l$; then $f_{\beta}^{R}=1$, corresponding to the unique tableau $s$ of shape $\lambda$ with critical subtableau of shape $[(m+1) l-1]$. The idempotent $p_{s}$ is evaluable by Lemma 1.7 and equivalent to $p_{t}$ by Lemmas $1.7(\mathrm{~b})$ and 1.8. Finally, suppose $\lambda_{2}>l$. Using the induction assumption for $z_{\beta}^{R}$ (which is the same as $z \frac{L}{\beta}$, where $\bar{\beta}$ is the reflection of $\beta$ in the $(m+1)$ st critical line), and arguing as above, we obtain $f_{\beta}^{R}$ mutually orthogonal, mutually equivalent evaluable idempotents dominated by $z_{\beta}^{R} z_{\lambda}$, and all equivalent to $p_{\tilde{r}} e_{n-1}$, where $\tilde{r}$ is some tableau of shape $\mu$ with critical subtableau of shape $[(m+1) l-1]$. But $p_{r}$ is equivalent to $p_{\tilde{r}}$ in $A_{n-2}$ by the induction assumption, so $p_{\tilde{r}} e_{n-1}$ is equivalent to $p_{r} e_{n-1}$ and hence to $p_{t}$ in $A_{n}$.

We now know that $z_{\lambda}$ is evaluable and has the required partition of unity; it remains only to verify the statement regarding equivalence of idempotents corresponding to regular tableaux. For two regular tableaux $s$ and $t$ both coming from the left or both from the right, equivalence of $p_{s}$ and $p_{t}$ follows from the equivalence of $p_{\left[s^{\prime}\right]}$ and $p_{\left[t^{\prime}\right]}$, which is given by the induction hypothesis, together with the equalities $p_{s}=z_{\lambda} p_{\left[s^{\prime}\right]}$ and $p_{t}=z_{\lambda} p_{\left[t^{\prime}\right]}$ and the centrality of $z_{\lambda}$. On the other hand, we already showed the equivalence of $p_{s}$ and $p_{t}$ for two particular regular tableaux $s$ and $t$, one coming from the left and one coming from the right.

Next we consider the case that $\lambda$ is non-critical. In case the predecessor $\beta$ of $\lambda$ is critical, both the existence of the required partition of unity and the statement regarding equivalence of idempotents follow from the induction statement applied to $z_{\alpha}^{L}$. Suppose next that $\alpha$ is critical. From the induction hypothesis applied to $z_{\alpha}=z_{\lambda}^{L} z_{\alpha}$, we obtain $f_{\alpha}$ mutually orthogonal mutually equivalent evaluable 
idempotents dominated by $z_{\lambda}^{L} z_{\alpha}$. Furthermore, using the induction hypothesis, Lemma 1.2 and Corollary 1.4, we see that each of these is equivalent to $p_{[t]}$, where $t$ is regular and has critical subtableau of shape $\alpha-[1,1]$. From the induction hypothesis applied to $z_{\beta}^{L}$, we obtain a collection of $f_{\beta}^{L}$ mutually equivalent, mutually orthogonal evaluable idempotents dominated by $z_{\beta}^{L} z_{\lambda}^{L}$, including $p_{[t]}$. This gives the required partition of unity and the evaluability of $z_{\lambda}^{L}$. Finally, the equivalence statement is obtained as before from the induction hypothesis, the centrality of $z_{\lambda}^{L}$ in $\left(z_{\alpha}+z_{\beta}^{L}\right) A_{n}\left(z_{\alpha}+z_{\beta}^{L}\right)$, and the equivalence, known from Corollary 1.4 , between $p_{[s]}$ and $p_{[t]}$ for two particular $s$ and $t$, with $s^{\prime} \in T(\alpha)$ and $t^{\prime} \in L(\beta)$.

The last case to consider is that the diagrams $\lambda, \alpha$, and $\beta$ are all non-critical. Let $t$ be any regular tableau of shape $\lambda$ such that $t^{\prime} \in L(\alpha)$, and $t^{\prime \prime}$ has shape $\mu=\lambda-[1,1]$. Set $s=\sigma_{n-1}(t)$, so that $s^{\prime} \in L(\beta)$. Then all of the idempotents $p_{[t]}, p_{\left[t^{\prime}\right]}, p_{[s]}$, and $p_{\left[s^{\prime}\right]}$ are evaluable by the induction hypothesis and Lemma 1.3(a), and $p_{[t]}$ and $p_{[s]}$ are equivalent by Lemma $1.3(\mathrm{~b})$. The induction assumption gives $f_{\alpha}^{L}$ mutually equivalent, mutually orthogonal evaluable idempotents dominated by $z_{\alpha}^{L} z_{\lambda}^{L}$, including $p_{[t]}$, and $f_{\beta}^{L}$ such idempotents dominated by $z_{\beta}^{L} z_{\lambda}^{L}$, including $p_{[s]}$. This gives the desired partition of unity and the evaluability of $z_{\lambda}^{L}$. The equivalence statement is proved as before.

THEOREM 2.2. (a) If $\lambda$ is a critical diagram, then $z_{\lambda}$ is evaluable at $q$, and is a minimal central idempotent in $A_{n}(q) d$. Furthermore $z_{\lambda} A_{n}(q) \cong \mathbf{M}_{f_{\lambda}}$.

Suppose now that $\lambda$ is a non-critical diagram. Then:

(b) $z_{\lambda}^{L}$ is evaluable at $q$.

(c) If $\lambda$ is to the left of the first critical line, then $z_{\lambda}^{L} A_{n} z_{\lambda}^{L}(q) \cong \mathbf{M}_{f_{\lambda}^{L}}$.

(d) Otherwise, $z_{\lambda}^{L} A_{n} z_{\lambda}^{L}(q) \cong\left\{\left[\begin{array}{ll}A & 0 \\ B & A\end{array}\right]: A, B \in \mathbf{M}_{f_{\lambda}^{L}}\right\}$.

Proof. From Proposition 2.1, we obtain for each critical diagram $\lambda$ an $f_{\lambda}$-by- $f_{\lambda}$ system of matrix units in $A_{n}(q)$ dominated by $z_{\lambda}(q)$, and for each non-critical diagram $\lambda$ an $f_{\lambda}^{L}$-by- $f_{\lambda}^{L}$ system dominated by $z_{\lambda}^{L}(q)$. If $\lambda$ is critical, then $\operatorname{dim}\left(z_{\lambda} A_{n}(q)\right)=f_{\lambda}^{2}$ by the principle of constancy of dimension, and therefore $z_{\lambda} A_{n}(q) \cong \mathbf{M}_{f_{\lambda}}$. Similarly, if $\lambda$ is to the left of the first critical line, then $z_{\lambda}^{L} A_{n} z_{\lambda}^{L}(q) \cong \mathrm{M}_{f_{\lambda}^{L}}$. If $\lambda$ is between the $m$ th and $(m+1)$ st critical lines for $m \geq 1$, then $\operatorname{dim}\left(z_{\lambda}^{L} A_{n} z_{\lambda}^{L}(q)\right)=2\left(f_{\lambda}^{L}\right)^{2}$, and the system of matrix units $\left\{u_{i j}\right\}$ 
in $z_{\lambda}^{L} A_{n} z_{\lambda}^{L}(q)$ can be chosen such that $u_{11}=p_{[t]}(q)$, where $t$ is a (necessarily evaluable) tableau of shape $\lambda$ with critical subtableau of shape $[m l-1]$. Now $n:=p_{[t]} e_{m l-1} p_{[t]}$ is a non-zero nilpotent of order 2 by Lemma 1.9. It follows that $N:=\sum u_{i 1} n u_{1 i}$ is a non-zero nilpotent of order 2 commuting with all $u_{i j}$. Thus the subalgebra of $z_{\lambda}^{L} A_{n} z_{\lambda}^{L}(q)$ generated by $N$ and the matrix units $u_{i j}$ is isomorphic to $\mathbf{M}_{f_{\lambda}^{L}} \otimes \mathbf{C}[x] /\left(x^{2}\right) \cong\left\{\left[\begin{array}{ll}A & 0 \\ B & A\end{array}\right]: A, B \in \mathbf{M}_{f_{\lambda}^{L}}\right\}$. Because of the dimension constraint, this is all of $z_{\lambda}^{L} A_{n} z_{\lambda}^{L}(q)$.

We can now finish the description of the blocks, the radical, and the maximal semisimple quotient of $A_{n}(q)$. We know that for a critical diagram $\lambda$ of size $n, z_{\lambda}(q)$ is a minimal central idempotent in $A_{n}(q)$, and $z_{\lambda} A_{n}(q) \cong \mathbf{M}_{f_{\lambda}^{L}}$. Now consider an orbit [ $\left.\lambda\right]$ of a non-critical diagram under the action of $A_{1}^{(1)}$. Note first that

$$
z_{[\lambda]}:=\sum_{\mu \in[\lambda]} z_{\mu}^{L}=\sum_{\mu \in[\lambda]} z_{\mu}
$$

is an evaluable central idempotent. We will show that $z_{[\lambda]} A_{n}(q)$ is a block of $A_{n}(q)$. If $\mu, \nu \in[\lambda]$ are adjacent (that is, if they are in adjacent fundamental intervals for the $A_{1}^{(1)}$ action), then $\operatorname{dim}\left(z_{\mu}^{L} A_{n} z_{\nu}^{L}(q)\right)$ $=f_{\mu}^{L} f_{\nu}^{L}$, while if $\mu$ and $\nu$ are not adjacent, then $z_{\mu}^{L} A_{n} z_{\nu}^{L}(q)=(0)$, by constancy of dimension. Furthermore, using Lemma 1.9 and the results of this section, one has for adjacent diagrams $\mu, \nu$,

$$
z_{\mu}^{L} A_{n} z_{\nu}^{L}(q) \operatorname{rad}\left(z_{\nu}^{L} A_{n} z_{\nu}^{L}(q)\right)=\operatorname{rad}\left(z_{\mu}^{L} A_{n} z_{\mu}^{L}(q)\right) z_{\mu}^{L} A_{n} z_{\nu}^{L}(q)=(0),
$$

and as a $\mathbf{M}_{f_{\mu}^{L}}-\mathbf{M}_{f_{\nu}^{L}}$ bimodule, $z_{\mu}^{L} A_{n} z_{\nu}^{L}(q)$ is isomorphic to the space of $f_{\mu}^{L}$-by- $f_{\nu}^{L}$ matrices. Moreover,

$$
z_{\mu}^{L} A_{n} z_{\nu}^{L} A_{n} z_{\mu}^{L}(q)=\operatorname{rad}\left(z_{\mu}^{L} A_{n} z_{\mu}^{L}(q)\right) .
$$

It follows that

$$
R_{[\lambda]}:=\bigoplus_{\substack{\mu, \nu \in[\lambda] \\ \mu, \nu \text { adjacent }}} z_{\mu}^{L} A_{n} z_{\nu}^{L}(q) \oplus \bigoplus_{\mu \in[\lambda]} \operatorname{rad}\left(z_{\mu}^{L} A_{n} z_{\mu}^{L}(q)\right)
$$

is a nilpotent ideal in $z_{[\lambda]} A_{n}(q)$. But the quotient is semisimple:

$$
z_{[\lambda]} A_{n}(q) / R_{[\lambda]} \cong \bigoplus_{\mu \in[\lambda]} z_{\mu}^{L} A_{n} z_{\mu}^{L}(q) / \operatorname{rad}\left(z_{\mu}^{L} A_{n} z_{\mu}^{L}(q)\right) \cong \bigoplus_{\mu \in[\lambda]} \mathbf{M}_{f_{\mu}^{L}},
$$

so in fact $R_{[\lambda]}$ is the radical of $z_{[\lambda]} A_{n}(q)$. Since the $z_{\mu}^{L}(q)$ are minimal central idempotents modulo the radical, it follows that any central 
idempotent in $z_{[\lambda]} A_{n}(q)$ is a sum of certain of these plus an element of $R_{[\lambda]}$. But one easily checks that no such element is a central idempotent, other than $z_{[\lambda]}(q)$. To summarize:

THEOREM 2.3. If $\lambda$ is a non-critical diagram, then $z_{[\lambda]}(q)$ is a minimal central idempotent in $A_{n}(q)$. The radical of $z_{[\lambda]} A_{n}(q)$ is nilpotent of exponent 3 and is spanned by the spaces $z_{\mu}^{L} A_{n} z_{\nu}^{L}(q)$ for pairs of adjacent diagrams $\mu, \nu$ in the orbit $[\lambda]$ and by the algebras $\operatorname{rad}\left(z_{\mu}^{L} A_{n} z_{\mu}^{L}(q)\right)$ for $\mu \in[\lambda]$. The maximal semisimple quotient of $z_{[\lambda]} A_{n}(q)$ is isomorphic to $\bigoplus_{\mu \in[\lambda]} \mathbf{M}_{f_{\mu}^{L}}$.

For each $\zeta \in \mathbf{C} \backslash\{-1\}$, one has a representation of $A_{n}(\zeta)$ on $\left(\mathbf{C}^{2}\right)^{\otimes n}$ defined as follows. Define $E(\zeta) \in \operatorname{End}\left(\mathbf{C}^{2} \otimes \mathbf{C}^{2}\right)$ by

$$
E(\zeta)=\frac{1}{1+\zeta}\left(\zeta \varepsilon_{11} \otimes \varepsilon_{22}+\varepsilon_{22} \otimes \varepsilon_{11}+\zeta \varepsilon_{12} \otimes \varepsilon_{21}+\varepsilon_{21} \otimes \varepsilon_{12}\right),
$$

and for $1 \leq i \leq n-1$, let $E_{i}(\zeta)=I \otimes \cdots \otimes I \otimes E(\zeta) \otimes \cdots \otimes I$, where $E(\zeta)$ acts in the $i$ th and $(i+1)$ st tensor places. Then $\pi_{\zeta}: e_{i} \mapsto E_{i}$ defines a representation of $A_{n}(\zeta)$. This representation was introduced by Temperley and Lieb and was later rediscovered by Pimsner and Popa [PP] in their study of the Jones index. The operator $E(\zeta)$ is a spectral idempotent for the $\check{R}$ matrix for the quantum universal enveloping algebra of $\operatorname{sl}(2)$ in its vector representation.

THEOREM 2.4. The representation $\pi_{\zeta}$ of $A_{n}(\zeta)$ on $\left(\mathbf{C}^{2}\right)^{\otimes n}$ is faithful for all $\zeta \neq-1$. The dimension of $\pi_{\zeta}\left(A_{n}(\zeta)\right)$ is the Catalan number $C_{n}$.

Proof. This is known for $\zeta$ not a root of unity, but we include a proof of this case as well. The faithfulness of the representation for $\zeta=1$ follows from the representation theory of the symmetric group on tensor space. If $p$ is any idempotent in the $\mathbf{C}(x)$-algebra $A_{n}$, then $p$ is evaluable at all but finitely many complex numbers and $\zeta \mapsto \pi_{\zeta}(p)$ is a continuous family of idempotents in $\operatorname{End}\left(\left(\mathbf{C}^{2}\right)^{\otimes n}\right)$ on the complement of this finite set, hence of constant rank. For all Young diagrams $\lambda$ with no more than two rows and for all $\zeta$ not a root of unity, $z_{\lambda}$ is evaluable at $\zeta$ and $z_{\lambda}(\zeta)$ is a minimal central idempotent in the semisimple algebra $A_{n}(\zeta)$. Since $\pi_{1}\left(z_{\lambda}\right)$ is nonzero, so is $\pi_{\zeta}\left(z_{\lambda}\right)$ for all such $\zeta$. Therefore $\pi_{\zeta}$ is faithful on $A_{n}(\zeta)$ for $\zeta$ not a root of unity.

Now consider the primitive $l$ th root of unity $q$. If $p$ is any idempotent in the $\mathbf{C}(x)$-algebra evaluable at $q$, then $p$ is also evaluable at 
$\zeta$ in a neighborhood of $q$. Because $\pi_{\zeta}$ is faithful and thus $\pi_{\zeta}(p) \neq 0$ for $\zeta$ in a deleted neighborhood of $q$, it follows by constancy of rank that $\pi_{q}(p) \neq 0$ as well. This implies that $\pi_{q}$ is faithful on a lifting of the maximal semisimple quotient of $A_{n}(q)$.

Let $\lambda$ be a non-critical diagram, $t$ a regular tableau of shape $\lambda$, and $n_{[t]}(q)$ a non-zero nilpotent in the two dimensional algebra $p_{[t]} A_{n} p_{[t]}(q)$. The proof of Lemma 1.9 shows that there is an evaluable minimal idempotent $f$ in $A_{N}$ for some $N>n$ such that $f n_{[t]} f(q)$ is a non-zero multiple of $f(q)$. The representations $\pi_{q}$ intertwine the usual imbeddings of $A_{n}(q)$ in $A_{N}(q)$ and of $\operatorname{End}\left(\left(\mathbf{C}^{2}\right)^{\otimes n}\right)$ in $\operatorname{End}\left(\left(\mathbf{C}^{2}\right)^{\otimes N}\right)$, so $\pi_{q}(f) \neq 0$ implies that $\pi_{q}\left(n_{[t]}\right) \neq 0$. Now for any non-zero element in $\operatorname{rad}\left(A_{n}(q)\right)$, the ideal generated by this element in $A_{n}(q)$ contains an element of the form $n_{[t]}$. Hence $\pi_{q}$ is faithful on $\operatorname{rad}\left(A_{n}(q)\right)$.

The statement regarding dimensions results from Proposition 0.1 .

It is also possible to give a short combinatorial proof of the faithfulness of the representation on tensor space which does not rely upon our structure theorem.

Acknowledgments. This work was partially supported by the following foundations: The United States NSF (FG and HW), the Sloan Foundation (HW), the Commemorative Association for the Japan World Exposition (1970) (FG and HW), and the Japan Society for the Promotion of Science (HW). FG thanks the University of California, San Diego for hospitality. Both authors thank the organizers of the RIMS 91 workshop at Kyoto University, where the manuscript was completed. We are grateful to Vic Camillo for helpful discussions.

\section{REFERENCES}

[B] R. Baxter, Exactly Solved Models in Statistical Mechanics, Academic Press, London, 1982.

[BW] J. Birman and $\mathrm{H}$. Wenzl, Braids, link polynomials and a new algebra, Trans. Amer. Math. Soc., 313 (1989), 249-273.

[Br] R. Brauer, On algebras which are connected with the semisimple continuous groups, Ann. of Math., 63 (1937), 854-872.

[H] P. Hoefsmit, Representations of Hecke algebras of finite groups with BN pairs of classical type, Thesis, University of British Columbia, 1974.

[J1] V. Jones, Index for subfactors, Invent. Math., 72 (1983), 1-25.

[J2] A polynomial invariant for knots via von Neumann algebras, Bull. Amer. Math. Soc. (N.S.), 12 (1985), 103-111. 
[L] R. Lickorish, Three-manifolds and the Temperley-Lieb algebra, Math. Ann., 290 (1991), 657-670.

[M] P. Martin, Potts Models and Related Problems in Statistical Mechanics, World Scientific, Singapore, 1991.

[PP] M. Pimsner and S. Popa, Entropy and index for subfactors, Ann. Sci. École Norm. Sup., $4^{\mathrm{e}}$ serie, 19 (1986), 57-106.

[RW] A. Ram and H. Wenzl, Matrix units for centralizer algebras, J. Algebra, 145 (1992), 378-395.

[SV] S. Stratila and D. Voiculescu, Representations of AF Algebras and the Group $U(\infty)$, Lecture Notes in Math., vol. 486, Springer-Verlag, Berlin and New York, 1975.

[TL] H. Temperley and E. Lieb, Relations between the 'percolation' and 'colouring' problem and other graph-theoretical problems associated with regular plane lattices: some exact results for the 'percolation' problem, Proc. Roy. Soc. London A, 322 (1971), 251-280.

[W1] H. Wenzl, On sequences of projections, C. R. Math. Rep. Acad. Sci. Canada IX, 1 (1987), 5-9.

[W2] _- Hecke algebras of type $A_{n}$ and subfactors, Invent. Math., 92 (1988), 349-383.

[W3] _ On the structure of Brauer's centralizer algebras, Ann. of Math., 128 (1988), 173-193.

[W4] _ Quantum groups and subfactors of type B , C , D , Comm. Math. Phys., 133 (1990), 383-432.

Received December 15, 1991. The first author was partially supported by the United States NSF. The second author was partially supported by the United States NSF and the Sloan Foundation.

UNIVERSITY OF IOWA

IowA CITY, IA 52242

E-mail address: goodman@math.uiowa.edu

AND

University of California, SAN Diego

LA Jolla, CA 92093

E-mail address: hwenzl@ucsd.edu 


\title{
PACIFIC JOURNAL OF MATHEMATICS
}

Founded by

\author{
E. F. BECKenBaCH (1906-1982) F. WolF (1904-1989)
}

\section{EDITORS}

Sun-Yung A. Chang

(Managing Editor)

University of California

Los Angeles, CA 90024-1555

chang@math.ucla.edu

F. Michael Christ

University of California

Los Angeles, CA 90024-1555

christ@math.ucla.edu

Herbert Clemens

University of Utah

Salt Lake City, UT 84112

clemens@math.utah.edu

\author{
R. FInN
}

THOMAS ENRIGHT

University of California, San Diego

La Jolla, CA 92093

tenright@ucsd.edu

Nicholas ERColani

University of Arizona

Tucson, AZ 85721

ercolani@math.arizona.edu

Stanford University

Stanford, CA 94305

finn@gauss.stanford.edu

VAUghan F. R. JoNES

University of California

Berkeley, CA 94720

vfr@math.berkeley.edu

\section{SUPPORTING INSTITUTIONS}

STEVEN KeRCKHOFF

Stanford University

Stanford, CA 94305

spk@gauss.stanford.edu

MARTIN ScharLemanN

University of California

Santa Barbara, CA 93106

mgscharl@henri.ucsb.edu

HAROLd STARK

University of California, San Diego La Jolla, CA 92093

V. S. VARADARAJAN

University of California

Los Angeles, CA 90024-1555

vsv@math.ucla.edu

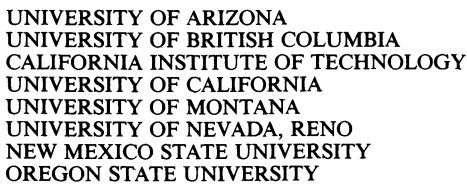

The Supporting Institutions listed above contribute to the cost of publication of this Journal, but they are not owners or publishers and have no responsibility for its content or policies.

Mathematical papers intended for publication in the Pacific Journal of Mathematics should be in typed form or offset-reproduced (not dittoed), double spaced with large margins. Please do not use built up fractions in the text of the manuscript. However, you may use them in the displayed equations. Underline Greek letters in red, German in green, and script in blue. The first paragraph must be capable of being used separately as a synopsis of the entire paper. In particular it should contain no bibliographic references. Please propose a heading for the odd numbered pages of less than 35 characters. Manuscripts, in triplicate, may be sent to any one of the editors. Please classify according to the 1991 Mathematics Subject Classification scheme which can be found in the December index volumes of Mathematical Reviews. Supply name and address of author to whom proofs should be sent. All other communications should be addressed to the managing editor, or Julie Honig, University of California, Los Angeles, California 90024-1555.

There are page-charges associated with articles appearing in the Pacific Journal of Mathematics. These charges are expected to be paid by the author's University, Government Agency or Company. If the author or authors do not have access to such Institutional support these charges are waived. Single authors will receive 75 free reprints; joint authors will receive a total of 100 free reprints. Additional copies may be obtained at cost in multiples of 50 .

The Pacific Journal of Mathematics (ISSN 0030-8730) is published monthly except for July and August. Regular subscription rate: $\$ 200.00$ a year (10 issues). Special rate: $\$ 100.00$ a year to individual members of supporting institutions.

Subscriptions, orders for numbers issued in the last three calendar years, and changes of address should be sent to Pacific Journal of Mathematics, P.O. Box 969, Carmel Valley, CA 93924, U.S.A. Old back numbers obtainable from Kraus Periodicals Co., Route 100, Millwood, NY 10546.

The Pacific Journal of Mathematics at P.O. Box 969, Carmel Valley, CA 93924 (ISSN 0030-8730) is published monthly except for July and August. Second-class postage paid at Carmel Valley, California 93924, and additional mailing offices. Postmaster: send address changes to Pacific Journal of Mathematics, P.O. Box 969, Carmel Valley, CA 93924.

PUBLISHED BY PACIFIC JOURNAL OF MATHEMATICS, A NON-PROFIT CORPORATION

This publication was typeset using $\mathcal{A} \mathcal{M S}-\mathrm{T}_{\mathrm{E}} \mathrm{X}$, the American Mathematical Society's $\mathrm{T}_{\mathrm{E}} \mathrm{X}$ macro system. Copyright (c) 1993 by Pacific Journal of Mathematics 


\section{PACIFIC JOURNAL OF MATHEMATICS}

Volume $161 \quad$ No. $2 \quad$ December 1993

On the method of constructing irreducible finite index subfactors of 201 Popa

FLORIN PETRE BOCA

Brownian motion and the heat semigroup on the path space of a

233 compact Lie group

JAY BARRY EPPERSON and TERRY M. LOHRENZ

Horizontal path spaces and Carnot-Carathéodory metrics

255

ZHONG GE

Biholomorphic convex mappings of ball in $\mathbb{C}^{n}$

SHENG GONG, SHI KUN WANG and QI HUANG YU

The Temperley-Lieb algebra at roots of unity

FREDERICK MiCHAEL GOODMAN and HANS WENZL

Jordan analogs of the Burnside and Jacobson density theorems

LUZIUS GRÜNENFELDER, M. OLMLADIČ and HEYDAR RADJAVI

Elliptic representations for $\mathrm{Sp}(2 n)$ and $\mathrm{SO}(n)$

REBECCA A. Herb

Reflexivity of subnormal operators

JOHN MCCARTHY

Knotting trivial knots and resulting knot types

KiMiHiko MOTEGI

Commutativity of selfadjoint operators

MitsURU UCHIYAMA

Correction to: "One-dimensional Nash groups" 